\section{DECREASE THE CHOLESTASIS INJURY IS VIA INHIBITION OF INTRINSIC PATHWAY WITH ENHANCED MITOCHONDRIAL BIOGENESIS}

\author{
M.-M. Tiao ${ }^{1,2}$, I.-F. Huang ${ }^{3}$, Y.-J. Yang ${ }^{4}$ \\ ${ }^{1}$ Pediatrics, Chang Gung Memorial Hospital- \\ Kaohsiung Medical Center, Chang Gung \\ University College of Medicine, ${ }^{2} \mathrm{Fu}$ Yin University, \\ ${ }^{3}$ Pediatrics, Kaohsiung Veterans General Hospital, \\ Kaohsiung, ${ }^{4}$ Pediatrics, National Cheng Kung \\ University and Hospital, Tianan, Taiwan R.O.C.
}

Background: Mitochondria are known to involve in cholestatic liver injury. The impaired mitochondrial biogenesis and intrinsic apoptosis pathway is activated associated with oxidative damage.

Aims: We further test the involvement of key mitochondrial apoptotic regulators in response to the early stage of corticosteroids on cholestasis and the possible protective mechanistic roles of steroid in this liver damage animal model.

Methods: Arat model of cholestasis was established by bile duct ligation (BDL), with simultaneously creation of the sham group receiving laparotomy without BDL, and dexamethasone (DEX) after BDL as a treatment. The expression of proteins involved in the activation of apoptotic pathways was analyzed by western blotting. Apoptosis of liver cells was examined by TUNEL stain.

Results: The apoptotic liver cells appeared in large amountsintheratliver by 72 hafterBDLin theapoptotic markers and could be recovered significantly after DEX treatment by $72 \mathrm{~h}$. A significant upregulation of liver peroxisome proliferators-activated receptor y coactivator-1 $\alpha$ and mitochondrial transcriptional factor A protein from 24 to $72 \mathrm{~h}$ was found in the DEX group. After DEX treatment, significant downregulated Bax, caspase 9 and caspase 3 at 24$72 \mathrm{~h}$, but not caspase $8, \mathrm{Bcl}_{2}$, Fas and Fas-Fas L complex in the liver homogenates of BDL rats was found. The activation of NFKB was inhibited by DEX treatment.

Conclusions: Our results indicate that early DEX treatment reverses impaired mitochondrial biogenesis within hours after BDL, via modulating of intrinsic apoptosis pathway. NFKB activity is involved in the process of liver cell damage.

\section{EFFICACY OF RIFAXIMIN FOR SIBO TREATMENT IN IBS CHILDREN}

V. Giorgio, V. D’ Andrea, P. Coccia, S. Filoni, C. Rendeli, C. Fundarò

Pediatrics, Catholic Unievrsity of the Sacred Heart, Rome, Italy

Background: Our previous study suggested a significant epidemiologic association between SIBO (Small Intestinal Bacterial Overgrowth) and IBS (Irritable Bowel Syndrome) in childhood. Rifaximin has already been successfully used in adults with SIBO and IBS.

Aim: To assess the SIBO prevalence and symptoms improvement in IBS children after treatment with Rifaximin.

Study design: 43 (mean age 9,5 ys) consecutive children affected by IBS according to Rome II criteria were enrolled. The control population $(n=28$, mean age $10 \mathrm{ys}$ ) consisted of healthy subjects without IBS symptoms. All subjects underwent lactulose/ methane breath test (LBT) to assess SIBO before and one month after the treatment with Rifaximin $600 \mathrm{mg}$ daily. All IBS pts filled out a Visual Analogic Scale (VAS) score to evaluate symptoms (abdominal pain, constipation, diarrhoea, bloating, flatulence) at time zero and 1 month after the treatment.

Results: The prevalence of abnormal LBT result was significantly higher in patients with IBS than controls $(65 \%, 28 / 43$ vs $7 \%, 2 / 28, p<0.05)$. One month after the treatment 18 pts had a negative LBT while 10 stayed positive $(64 \%, 18 / 28$ vs $36 \%, 10 / 28, p<$ 0.05). In IBS pts VAS score was significantly higher in SIBO positive than SIBO negative children. One month after treatment VAS score was significantly lower in eradicated than non-eradicated pts.

Conclusions: Rifaximin is safe and seems to be effective in SIBO eradication and symptoms improvement of IBS pts. Other placebo-controlled interventional studies to treat bacterial overgrowth are warranted to verify the real impact of SIBO on IBS symptoms. 